\title{
Community-based Hazard Warnings in Rural Sri Lanka: Performance of Alerting and Notification in a Last-Mile Message Relay
}

\author{
Gordon Gow, PhD \\ Assistant Professor \\ Graduate Program in Communication and Technology, \\ Faculty of Extension, University of Alberta, \\ Edmonton, Alberta, Canada T6G 2T4 \\ Tele: +1 780710 4673, Fax: +1 7183953587 \\ Email: ggow@ualberta.ca
}

\author{
Peter Anderson \\ Associate Professor \\ Director - Telematics Research Lab, \\ School of Communication, Simon Fraser University, \\ 8888 University Drive, Burnaby, B.C. Canada V5A 1 S6 \\ Tele: +1 778782492 \\ Email: anderson@sfu.ca
}

\author{
Nuwan Waidyanatha \\ Project Manager - Last-Mile Hazard Warning System \\ LIRNEasia, 12 Balcombe Place, Colombo 08, Sri Lanka \\ Tele: +94 773710 394, Fax: +94 112675212 \\ Email: waidyanatha@lirne.net
}

\begin{abstract}
The aim of the Last-Mile Hazard Warning System (LM-HWS) is to deploy and assess various alert and notification technologies intended to reduce the vulnerability of local communities to natural and manmade hazards in rural Sri Lanka. The project adopts an "all-hazards, all-media" approach designed around a set of five wireless communication technologies [2]. The pilot project involved deployment, training, and field-testing of the technologies, in various combinations, across 32 tsunami-affected villages, using Common Alerting Protocol for data interchange with content provided in three languages (English, Sinhalese and Tamil). This paper reports on findings from a series of field tests conducted in Sri Lanka to compare the reliability of the five 'last-mile' devices with their relative effectiveness in terms of alert and notification capabilities. Findings indicate that overall effectiveness of the alert and notification system is enhanced when a village is equipped with a technology combination that enhances complementary redundancy in reliability and effectiveness. Further implications of these findings for planning and future research are discussed.
\end{abstract}

\section{INTRODUCTION}

In December 2005, LIRNEAsia, an ICT policy and reform research organization, initiated a research project to evaluate the "last-mile" communication component of an all-hazards warning system for Sri Lanka. The "HazInfo" project was funded by Canada's International Development Research Centre (IDRC). Research design was based on recommendations contained in a concept paper developed following the 2004 tsunami. $^{1}$

\footnotetext{
${ }^{1}$ Samarajiva, Rohan, Knight-John, Malathy, Anderson, Peter, Zainudeen, Ayesha et al. (2005, March 17). National Early Warning System Sri Lanka:
}

The primary objective of the research project ${ }^{2}$ was to evaluate five wireless ICTs deployed in varied conditions for their suitability in the 'last-mile' of a national disaster warning system for Sri Lanka and possibly by extension to other developing countries. This paper compares a subset of indicators for evaluating system design and performance; namely, the reliability of the wireless technologies for delivering alert message to local villages compared against their relative effectiveness in terms of alert and notification functions. Such a comparison is important in terms of understanding the trade-off between deploying highly reliable systems versus those devices that are best able to alert and communicate hazard information to local first responders. It is anticipated that an improved understanding of these factors can lead to better investment decisions in warning technologies and improved design of warning messages using Common Alerting Protocol.

\section{LM-HWS ARCHITECTURE}

The HazInfo project involves a non-government organization (NGO) Sarvodaya ${ }^{3}$ and is established on a governance

A Participatory Concept Paper for the Design of an Effective All Hazard Public Warning System Version 2.1, LIRNEasia and Vanguard Foundation, 2006. Available http://www.lirneasia.net/projects/completedprojects/national-early-warning-system/

${ }^{2}$ Research Project: Evaluating Last-Mile Hazard Information Dissemination (HazInfo). 2006. Available

http://www.lirneasia.net/projects/current-projects/evaluatinglast-mile-hazard-information-dissemination-hazinfo/

${ }^{3}$ The Lanka Jatika Sarvodaya Shramadana Sangamaya (Sarvodaya) is Sri Lanka's largest and most broadly embedded people's organization, with a network covering: 15,000 villages; http://www.sarvodaya.org/about. The 32 Selected Sarvodaya Communities were affected by the December 2004 Indian Ocean Tsunami and uniformly represent the 10 Tsunami affected 
structure whereby this organization provides project oversight, training, and operates a Hazard Information Hub (HIH) for the monitoring of hazard threats and dissemination of warning messages to local communities within the Sarvodaya network of villages. Each of the 32 participating communities varies in size from 150 - 1000 households.

The LM-HWS architecture depicted in Fig. 1 complements the traditional public alerting system design usually established by local and/or national governments. A traditional public alerting system issues warnings directly to communities via broadcast media such as television and radio, or through designated public address (PA) systems. By contrast, the LM-HWS project architecture establishes a closed user group of first responders, who are equipped with addressable wireless devices for receiving bulletins issued from Sarvodaya's Hazard Information Hub.

A simplified information flow for the LM-HWS is as follows: staff members at the HIH monitor hazard events around-the-clock using the Internet. When a potential threat is detected, the HIH activates its Emergency Response Plan (ERP) by issuing a message to the $n$-number of communities at risk using a combination of wireless ICTs to reach local first responders (denoted by the arrow between the HIH and ICT-G blocks in Fig. 1). Each community has assigned a person or persons to be responsible for managing the wireless device and monitoring it for incoming warning messages. This person has received training from Sarvodaya and is designated as a community ICT-Guardian (ICT-G). When the ICT-G receives a warning message at the HIH, they are responsible for activating the community-level ERP. The community response will vary depending on the content of the message, including its priority level. During activation, the ICT-G informs the $m$-number of ERP Coordinators (ERP-C), consisting of a First-Aid team, Evacuation team, Security team, and Message Dissemination team. The Message Dissemination team then relays the message village-wide through various methods, including as word-ofmouth, ringing local temple bells, loudspeaker, and so forth.

Message content is encoded using Common Alerting Protocol (CAP), an open source data interchange standard that includes numerous fields intended to provide consistent

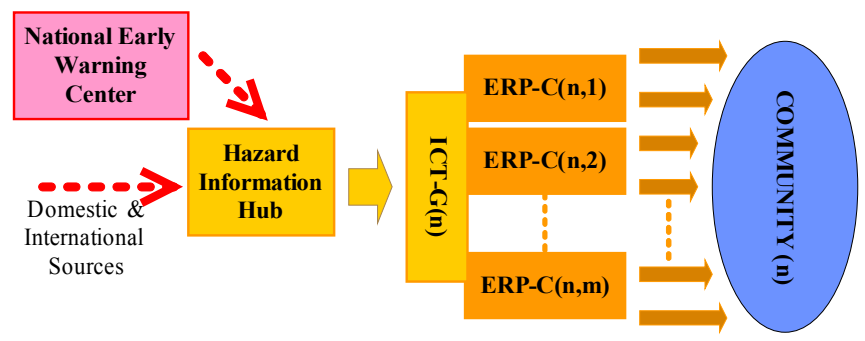

Figure 1 - end-to-end hazard information communication architecture of the LM-HWS; where messages received at the $\mathrm{HIH}$ are relayed to the villages.

Coastal Districts of Sri Lanka. These Communities also represent the different ethnic communities in the country. and complete messages across different technologies. ${ }^{4}$ The implementation of CAP in the LM-HWS is an important aspect of the project because it is key in establishing an "allmedia" warning capability.

\section{EVALUATING THE LM-HWS}

The research proposal of this project defined six specific research parameters for assessment: reliability of different wireless devices for transmitting messages, effectiveness of devices for alert and notification, impact of the technology on community organizational structure, effectiveness of the training regime, gender specific concerns, and integration of the wireless technology into the daily activities of the villages. This paper will focus on the first two parameters concerning the reliability and effectiveness of wireless technologies for providing hazard warnings to villages participating in the LM-HWS project.

LM-HWS assessed five wireless technologies, selected for their diverse communication paths and different features: Addressable Satellite Radios for Emergency Alerts (AREA), a specialized Remote Alarm Device (RAD), Mobile Handheld Phones (MOP), Wireless Fixed Phones (FXP), and Very Small Aperture Terminal (VSAT) coupled with a Personal Computer. The technologies were deployed in communities in a heterogeneous configuration. The research team also acknowledged the importance of incorporating "bidirectional" capabilities at the village level so that devices could provide communities with means to inquire of situations and inform local hazards to the Sarvodaya HIH (upstream communication). The AREA unit, described in section III (A) of this paper was the only device that was limited to downstream communication. Therefore, this particular equipment was married with one of the other 4 equipments to form an ICT where the coupled configuration: AREA+MOP, AREA+FXP, AREA+RAD, and AREA+VSAT would have bi-directional capabilities.

\section{CALCULATING RELIABILITY OF ALERTING PROCESSES}

The basic question governing the reliability measure is "did the ICT work on the day of the live-exercise?" Reliability, denoted by $R$ can be measured in at least two aspects: certainty and efficiency; denoted by $R_{c}$ and $R_{e}$ respectively. Whereas certainty refers to the operational state of a device on the day of the exercise, efficiency measures the time taken to complete the transmission of a message in relation to the anticipated hazard risk (i.e., will the message be received with enough advance warning to take action?).

\footnotetext{
${ }^{4}$ Botterell, Art and Addams-Moring, Ronja. (2007). Public warning in the networked age: open standards to the rescue. Communications of the ACM, $30(7), 59-60$
} 
In some situations the ICT failed on the day of the exercise and was given a reliability score of 0 in terms of certainty. For the purpose of this project, the calculation of reliability, in terms of certainty, is defined as in the formula:

$$
R_{c}= \begin{cases}1 & \text { device can certainly receive alerts } \\ 0 & \text { otherwise }\end{cases}
$$

In conventional analysis, probability of reliability requires a large amount of data gathered from multiple trials, a luxury the LM-HWS does not have. Therefore, the project used the methodology proposed in [6], which provides means to quantify $R_{e}$ using Mean-Time-To-Failure (MTTF) with a single set of data. MTTF is a measure of expected time where the process will fail. Hence, reliability, in terms of efficiency, is defined as $R_{e}=1-M T T F$. The reliability: $R_{e}$ measure would consider the aggregate of the time it took the $\mathrm{HIH}$ to transmit and alert message to the ICT-G and the time an ERP-C spent on activating the ERP at the community level (see Fig. 1).

Let us denote the period of completing the LM-HWS processes with variable $\bar{T}$. An assumption is made that these processes must be completed by a benchmark time; i.e. expected time $E(\bar{T})$. Define the "hazard-duration" to be the difference between the hazard initiating time and the hazard impacting time, denoted by the variable $T$. The reliability: $R_{e}$ of an LM-HWS exercise is calculated using the following formula:

$$
R_{e}=1-\left(\frac{\bar{T}-E(\bar{T})}{T}\right)
$$

when $\bar{T}>E(\bar{T})$; otherwise $R_{e}=1$ because the process was completed prior to the benchmark time. Similarly, the other extreme is if $\bar{T}>T$, then $R_{e}=0$ because the time has surpassed the maximum allowable time; i.e. hazard-duration. Since this research is first of its kind and the live-exercises conducted in Sri Lanka was the initial set of tests. Therefore, an assumption was made to set the benchmark value to the best case scenario $E(\bar{T})=0$. In the future $E(\bar{T})$ can be estimated using the previous set of data; i.e. the data collected during the live-exercise conducted between November 2006 and May 2007.

Using (1) and (2), the overall reliability $R$ of the wireless ICT in a LM-HWS is computed as functions of certainty and efficiency; where:

$$
R=R_{c} \times R_{e}
$$

\section{EFFECTIVENESS OF DEVICE ALERTING CAPABILITIES}

Effectiveness was measured as a function of a set of discrete parameters. The project has defined 11 such discrete parameters: language diversity, full CAP capability, audio and text medium availability, bi-directionality, total cost of ownership, DC power consumption, daily utilization, acknowledgment of message receipt, active alerting functionality, weight of wireless ICT, and signal coverage. Each parameter is denoted by a literal $q_{1}, q_{2}, \ldots, q_{11}$. A "Liken" type rating is used to obtain a real valued score between 0 and 1 for each literal, which is denoted by $G\left(q_{i}\right)$ for $i=1 \ldots 11$. A conjunction of the literals $q_{1} \wedge q_{2} \wedge \ldots \wedge q_{11}$ defines the design requirement for an effective ICT in a LMHWS. Hence, a single score for the effectiveness $G$ of the deployed wireless ICT configuration is obtained by multiplying the real valued score of all the literals, as stated in equation (4).

$G=G\left(q_{1} \wedge q_{2} \wedge \ldots \wedge q_{11}\right)=G\left(q_{1}\right) \times G\left(q_{2}\right) \times \ldots \times G\left(q_{11}\right)$

A subset of the mentioned parameters (language diversity, full CAP capability, and audio/text medium availability), which defines "CAP Compliance", is already discussed in [9]. Therefore, this paper chooses to discuss an equally important feature, which is the "Alerting" capability of wireless ICT terminal devices. The project defines Alerting to be a function governed by the clique of parameter: message receipt acknowledgment, active alert function, and geographical signal coverage. The research acknowledges that simply issuing the message from the $\mathrm{HIH}$ alone is inadequate. The Alerting function is complete if the message is received by the ICT-G and an acknowledgment message is returned via the same or alternate path to the Sender at the Message-Relay (HIH).

\section{A. Active Alert Function}

The active alert function parameter concerns the ability of the device to get the attention of the recipient ICT-G with one or more features: sounding-sirens, flashing-lights, or mechanical-vibrations. Ideally, an alert feature should be capable of working on each of the sensory domains: aural, visual, and tactile (e.g, vibration). The ICT terminal device scores 1.0 if it has features in all three domains; scores 0.95 if it has both audible and visual features because this combination does not require the device to be attached to the ICT-G in any way; scores 0.85 if it sounds a siren and vibrates; scores 0.70 if it sounds a audible siren only; scores 0.50 if it has both visual and tactile features; scores 0.25 if it has either a visual or tactile feature only; scores 0 if it has no active alert feature. A sigmoid type exponential scaling function is applied because, relative to light, an audible sound is not constrained as much by indoor environments 
and is also far more effective in waking up a sleeping ICT-G. Therefore, ICT terminals with audible sound are given a much higher score relative to ICT Terminals with visual and tactile sensory only. Similarly a tactile sensory features, which requires the device to be attached to a person is preceded by a flashing light. A combination of the sensory features would provide redundancy and as a result be highly effective. The defined enumeration methodology is a very primitive way of assessing the Active Alerting Function. It is imperative that a pragmatic and accurate methodology is developed using Human-Computer-Interaction (HCI) or Man-Machine-Interaction (MMI) Theory.

\section{B. Message Receipt Acknowledgment}

This is a vital feature for the ICT-G to inform the Senders that the message was received and understood and not just delivered to the terminal device. In the context of Alerting the acknowledgment must be initiated by a human. Hence, the devices must have a method in place to send an acknowledgment as soon as the ICT-G reads or listens and accepts the message. The device would score 1.0 if the ICT Terminal has a feature for acknowledgment and scores 0 otherwise. This parameter does not address the importance of the time it takes, relative to the hazard-duration $T$, for the staff members at the $\mathrm{HIH}$ to receive an acknowledgment in the context of certainty (reliability) of message receipt. This parameter simply addresses a yes or no answer to whether the ICT has the option of sending an acknowledgment to the sender. As mentioned in section III, the AREA Terminal does not have a return path or upstream channel to be used for sending an acknowledgment to the $\mathrm{HIH}$; whereas all the other devices tested in the project do have means to issue an acknowledgment.

\section{Geographical Signal Coverage}

Signal coverage measures the effectiveness of reaching an ICT-G recipient at various times of day and is intended to reflect the variability between mobile, nomadic, and fixed wireless devices. Signal strength is important for the Alerting feature because if the signal is weak then some data can get lost. As a result the terminal device may not get the full bit string, causing failure to activate some of its active alerting function sensory features that are triggered by designated bits in the data string. Wireless signal is usually measured as a function of the power of the signal in decibels (dB) then referenced to 1 mill watt $(\mathrm{dBm})$. The signal strength was measured at the ICT-Gs home or the location where equipment was installed. A GSM mobile phone can function on -104 to $-47 \mathrm{dBm}$ range, Satellites operates on -127 to $-60 \mathrm{dBm}$ range, and CDMA phone operates on -106 to $-48 \mathrm{dBm}$ range. All the devices used in the project display their signal strength using illumination of histogram bars (or LEDs). Given the device has a total of $y_{\max }$ bars, at a particular location such as the ICT-G's home, the device may have $y \leq y_{\max }$ number of bars illuminated; where $y$ is a real number. For example, a particular terminal device may have the bars color coded; where a green bar implies full strength, amber bar implies half strength, and red bar implies zero strength. With such a color coding, a device with a maximum of 5 bars having 2 green bars, 1 amber bar, and 2 red bars illuminated may imply $y=2.5$. For a particular application of the ICT (e.g., application of sending and receiving SMS) to work properly the service provider can specify a minimum requirement for the signal strength (such as 1 bar). Let us denote this minimum strength by $y_{\text {min }}$. The device is given a score based on the signal strength indicated by the bars by applying the following formula:

$$
G(\text { signal strength })=\left\{\begin{array}{cl}
1 / 1+e^{y_{\min }-y} & , y \geq y_{\min } \\
0 & , y<y_{\min }
\end{array}\right.
$$

The exponential function defined in (5) will give a score between 0 and 1 . When $y=y_{\min }$ the score will be 0.5 , implying that with the terminal device indicating minimum signal strength there is only a $50 \%$ chance that the alert message will come through completely. As the number of bar $y>y_{\min }$ increase the score will reach a value of 1 at an exponential rate. Similarly as the number of bars decrease below the minimum number of bars needed the probability of receiving the alert drops below 50\%. The calculation takes in to consideration that $y_{\max }-y_{\min } \approx 5$ because all of the ICT terminal devices tested in this project had either a 5 bar or 7 bar signal strength display; where all of the devices with 5 bars required approximately minimum application specific requirement of 2 bars and the AREA with a 7 bar display required approximately a 3 bar minimum requirement. It is evident that the signal strength scoring equation (5) has to be refined and generalized for all ICT terminal devices. For the purpose of this research equation (5) was sufficient.

\section{EXPECTED PERFORMANCE OF THE DEVICES}

\section{A. Addressable Radio for Emergency Alerts}

AREA is a class of WorldSpace ${ }^{5}$ Systems that is designed to be used in Disaster Warning, Recovery, and Response. Global Positioning System (GPS) technology incorporated into the radio set, along with the unique code assigned to every satellite radio receiver, allows for hazard warnings to be issued to sets that are within a vulnerable area or just to radio sets with specific assigned codes. WorldSpace has the capacity to easily reach widely dispersed geographic locations and even in areas where there is no telephone connectivity. The limitless reach is further augmented by alternative power-supply solutions that address the lack of connectivity to public electrical power grids as well.

\footnotetext{
${ }^{5}$ WorldSpace Addressable Satellite Radio url - $\underline{\text { http://www.worldspace.com }}$
} 
This solution communicates UDP/IP data packets channeled up to a Satellite through X-Band and down to the AREA devices through L-Band communication pipes. The receiver, called DAMB-R2, is a low-memory radio with a small display and a limited processing power. DAMB-R2 features dual channel reception with one of the channels designated as a data channel called OAAC (Over Air Activation Channel). Through the OAAC channel the alert provider can send an alert message. DAMB-R2 has the capability to monitor the alert, validate the message and perform the specified action. The action could range from activating a relay for a siren, turning on/switch to a WorldSpace channel for audio messages and displaying text regarding the alert. An optional USB connectible device is available to provide expanded message display capability [7].

In terms of performance, the AREA device should have good reliability as the system features battery backup and a relatively simple user interface, although some problems with antenna positioning were noted. At the HIH, the AREA system has a stable platform for composing and transmitting messages and a dedicated network for uplink, reducing concerns about delays in transmission. The AREA devices also have active alert features composed of a visual and audible siren system, as well as a limited text display for message content. With good signal coverage throughout Sri Lanka and these features, the AREA devices were expected to perform with relatively high effectiveness despite the fact that they do not have a message acknowledgment feature.

\section{B. Remote Alarm Device}

RAD is based entirely on widely available mobile communications technologies Short Messages (SMS) and Cell Broadcast Messages (CBM). SMS Based alerting is used to activate selected or individual RADs, while the CBM is used to activate all RADs. These terminal devices are standalone units that incorporate remotely activated alarms, flashing lights, a broadcast FM radio receiver to be turned off or on as directed by the message, the displaying of the SMS messages on LCD panel, a self-test button, message acknowledgment and a dynamic hotline GSM call-back feature for user to acquire additional information. Five push button switches labeled as Call, Ack, LCD, Test, and Radio control the operational states of the device. The GSM Alarm Device is a product of the University of Moratuwa Dialog 6 Communication Research Lab [4].

The microcontroller and the GSM module are the key components of the Alarm Device. The microcontroller houses a multitude of peripheral devices such as internal program flash memory, Data Memory, general purpose I/O, and USARTS. Once in operation, the GSM module listens

\footnotetext{
${ }^{6}$ Dialog Telekom url on the Disaster and Emergency Warning Network http://www.dialog.lk/en/corporate/cr/ourapproach/innovationinclusion/dewn. $\underline{\mathrm{html}}$
}

for any incoming SMS messages or CBMs. CBM-based warning messages will be broadcast on a predetermined dedicated logical broadcast channel. Upon the reception of a CBM or an SMS, a notification will be sent by the GSM module to the microcontroller. The microcontroller in turn will read and processes the message. If the message is from an authorized source (in case of SMS) and conforms to a given format the Alarm Device will be triggered. The RAD is designed to power up from the mains supply but is equipped with a seven hour back up battery as a secondary power source.

HazInfo project was the first to field test the RADs. Hence usability and effectiveness of the devices were questionable from the beginning. Having disregarded the issue faced by congestion in SMS applications the units were found to be exceptionally reliable during Lab tests. The external turningindicator type flashing light used in vehicles and 40watt speaker ranked the RAD to be the unit to have the most forceful active alerting functions. The 160 character limited display designed for English text messaging only established the device to have very low effectiveness. Since the GSM signals cover only 60 percent of Sri Lanka the units would be limited to locations with good signal coverage. The sets had absolutely no value addition to integrating them in to the village daily life, reducing the effectiveness scores further.

\section{Mobile Phones}

The research used Nokia 6600 MOP that are powered by a $104 \mathrm{MHz}$ ARM processor, and is based around Symbian's Series 60 platform. Microimage ${ }^{7}$ developed a J2ME applet that sits on Symbian Operating System. The MOPs are activated by a SMS sent from an Internet Application that can be configured to send alerts to all or a group of MOP handsets. The GSM Java enabled SMS mobile phones receive text alerts in Sinhala, Tamil and English, sounds an alarm, and has a hotline GSM call-back feature.

Given that the MOP had a java based client software that fulfilled all three features of the active alerting function, as well as text messaging in all three national languages the devices was expected to perform the best with very high effectiveness. Since SMS messaging requires less signal strength opposed to voice the MOP based solution was, further, trusted to be highly effective. The ubiquitous technology with a device that can be alongside the ICT-G at all times and could be easily integrated in to their daily village life would prove to be most reliable device of the lot.

\section{Wireless Fixed Phones}

CDMA FXP was used in the research, a Sri Lanka Telecom ${ }^{8}$ solution called City Link for telephony mainly for Rural Sri Lanka. The phone sets also provide 1xRTT capabilities with

\footnotetext{
${ }^{7}$ Microimage website - http://www.microimage.com

${ }^{8}$ Sri Lanka Telecom website - http://www.slt.lk
} 
Voice, SMS, Fax, and Internet connectivity (54Kbps), which transmit on the $8.5 \mathrm{KHz}$ and $18.5 \mathrm{KHz}$ frequency spectrums. CDMA Fixed Wireless Phones with built-in speakerphones to provide voice communication via the public switched FXPs.

The alerting for was a simple voice call, which would allow for an alert message to be voiced in any language. However, the language diversity of the staff at the HIH would limit the multilingual capabilities. With CDMA coverage spanning all most the entire country it would be highly reliable solution. Loud ring tone is a definite attention getter. However, the nomadic terminal device requires that the ICT-G be in close proximity. Also if the call is received by the ICT-G, then acknowledgment is instantaneous.

\section{E. Very Small Aperture Terminals}

The VSATs installed by Innovative Technologies ${ }^{9}$ the project operate on the C-Band and used IP for communications. Financial constraints forced for shared network using shared DVB (Digital Video Broadcast) for receiving. The Hazard Information Hub, serving as the central gateway, interconnected with earth stations in remote locations. The topology allowed for any VSAT station to serve as the central gateway. The Antenna used at the HIH was a Channel Master $^{10} 2.4 \mathrm{M}$ C-Band linear antenna. The other ground stations used 1.8M C-Band linear antennas. Each antenna was aligned with the AsiaSat II satellite. All antennas were fitted with an Agilis ${ }^{11} \mathrm{C}$-Band BUC (Block Up-Converter) and LNB (Low Noise Block, also known as Low Noise Converter). Two co-axial cables running from the BUCs and LNBs are connected to iDirect 3000 series modems/routers and local network switches.

Alerts were issued and received with the use of the Internet Public Alerting System (IPAS). IPAS Message Clients were tested on Personal Computers. The PCs were preloaded with Windows OS. The software is configured to be activated upon PC power up. Thereafter, the IPAS Client prompts the user to login. After logging in, the IPAS Client runs as a service in the background and is on standby listening to a dedicated Communication port for any incoming alerts messages. Alerts are sent through a Web Services based text messaging technique. When an IPAS Alert is received, if configured to Siren On, immediately sends a siren sound to the PC speakers and a Text Display window pops up on the screen with an Alert Message. After 10 seconds the sound is turned off. The end-user can cancel the Text Display and set the IPAS Client back on standby mode. If the user does not

\footnotetext{
${ }^{9}$ Innovative Technologies is a Sri Lankan company run by Warnakulasuriya et al (warnasl@yahoo.com), an Engineer who specializes in manufacturing antennas and installs satellite communication solutions in the Asia region.

${ }^{10}$ Channel Master website - http://www.channelmaster.com and specs of antenna can be obtained here -- http://www.esa-com.com/shop/type243-CINTELSATapproved.pdf

${ }^{11}$ Agilis website -- http://www.agilis.com and specs of the ALB 180 series BUC can be found here -- http://www.mwsatcom.ru/agilis/BUC_s.pdf
}

wish to receive alerts then the user can log out. Also if the $\mathrm{PC}$ is being powered down the operating system will force IPAS to $\log$ out before turning the PC Power off. IPAS software was developed by Solana Networks ${ }^{12}$.

The alerting solution was limited to English text messaging and the need for costly infrastructure insinuating poor effectiveness. However, the strong foot print of AsiaSat II would prove the solution to be efficient. Since the solution requires a computer to be on and manned it is uncertain that an alert would be received at a given time.

\section{ACTUAL PERFORMANCE OF THE DEVICES}

Observations in this paragraph refer to Fig. 2. The analysis clearly indicates that the AREA + MOP and AREA + FXP ICT deployments (configurations) to be the most suitable in a LM-HWS. The AREA + RAD shows to be equally reliable, mainly because the high reliability of the stand alone AREA unit. However, the AREA + RAD marriage has a very low effectiveness because the very low effectiveness of the stand alone RAD drags effectiveness of the coupled configuration down. There is no reliability score for the stand alone RAD because none of the communities were deployed with stand alone RADs. AREA also has a very low effectiveness since the text alerts were issued in English language and the communities found these units to be a little complex to operate as they were novel items and were not known to them as well as the market saturated GSM MOPs and CDMA FXPs. Stand alone MOPs and FXPs show a low effectiveness because the weakness in signal strength of GSM and CDMA footprints in Sri Lanka as well as the limitations of the devices constraining full and complete messaging capabilities. Reliability of the MOP was low as a result of the communities accidentally deleting the Java applet or the SMS that triggered the applet was not coming

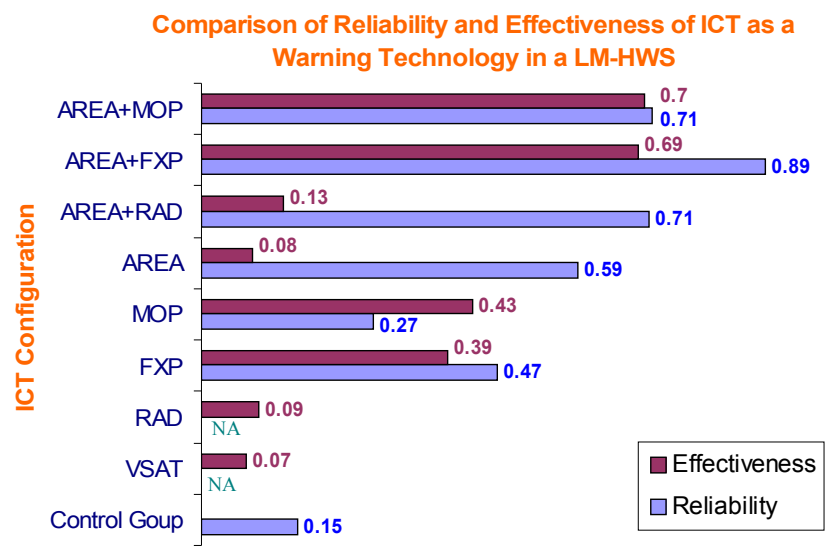

Figure 2 - Illustrates the comparison of Reliability (blue bars) and effectiveness (purple bars) of the ICT deployments. 'NA' implies Not Applicable.

\footnotetext{
${ }^{12}$ Solana Networks Internet Public Alerting System http://www.solananetworks.com
} 
through. Therefore, the particular MOP ICT failed on many occasions on the day of the exercise. As a result of the conflict situation in the North and East of the Island the Military had instructed all commercial wireless operators to shut off cells in the particular battle zones. This in turn affected the outcome of the live-exercises of communities deployed with MOPs and FXPs. However, the unidirectional AREA satellite based sets functioned in the war-zones when the terrestrial technologies were shut off. Project was unable to conduct live-exercises in communities with VSATs due delays in deployment of the earth stations in the respective communities. "Control Group", on the Y-axis of the bar graphs in Fig. 2, indicate communities that were used as control samples and were not given any LM-HWS ICTs. However, in all situations the control group communities had an organizational structure to make an alliance with another neighboring community who had received an LM-HWS ICT. The ally-community with LM-HWS ICT would relay the alert message to the control community over existing technologies such with the use of personal telephones.

\section{COMPLEMENTARY REDUNDANCY}

Test initial results show all devices to take less than one minute to push the alert messages to the end user devices but overall scores for reliability varied considerably. Effectiveness also varied widely across devices highlighting several user training concerns as well as some unforeseen difficulties implementing and maintaining the devices. Looking at the overall picture, the AREA performed very reliably in part because the footprint of the WorldSpace AsiaStar is strong in Sri Lanka. However, the AREA was somewhat less effective than initially anticipated because of limitations of text display and user friendliness issues. On the other hand, the MOP provide to be a very effective means of conveying messages to the ICT-G, despite the fact that its reliability score proved to be lower than anticipated. This is in part because terrestrial wireless telephony networks have not fully penetrated the rural areas of Sri Lanka and cover only about 70 percent of the Island. However, when considering combined performance of the devices, the high reliability of the AREA device with the high effectiveness score of the MOP provided what we might term "complementary redundancy" with the two devices working well together in balancing reliability with effectiveness.

\section{CONCLUSION}

Results from initial field tests comparing reliability with effectiveness suggest that appropriate combinations of wireless technologies will provide the best performance if they exhibit complementary redundancy. These results have a number of implications for emergency planners. First, planners should consider the deployment of multiple devices with the aim of achieving complementary redundancy in reliability and effectiveness. Second, planners should adopt the Common Alerting Protocol because of its ability to support the goal of complementary redundancy by providing consistent and complete messaging across multiple devices. Third, that research is needed to further refine the reliability and effectiveness measures and to undertake more field testing in order to contribute to best practices in planning and deploying combinations of wireless devices for hazard warning.

\section{REFERENCE}

[1] Anderson, Peter (2006) Last of the Mile Warning System ICT Assessment and Community Simulation Observation Report, LIRNEasia HazInfo Project [available from author - anderson@sfu.ca]

[2] A. Beresnev "Measuring Devices and Methods for Analysis of Coverage Area in GSM Cellular Systems, IEEE Conference on Electron Devices and Materials, Thomsk State University of Control Systems and Radioelecronics, Tomsk, Rassia. Paper can be obtained from authorsan@.tcc.tomsk.ru (Published)

[3] J. Chesterfield, P. Rodriguez, (2003). DeltaCast: Effetient File Reconciliation in Wireless Broadcast systems. Paper available here -http://research.microsoft.com/ pablo/papers/deltacast_mobisys.pdf

[4] Dias, Dileeka, Jayasinghe, Gamini, Fahmy, Farazy, Galaweera, Nuwan (ICIIS 2006). A GSM Alarm Device for Disaster Early Warning. Proceedings of the First International Conference on Industrial and Information Systems, Peradeniya, Sri Lanka. (Published)

[5] G. Gow, (2007 March/April). Implementing Common Alerting Protocol for Hazard Warning in Sri Lanka, Journal of Emergency Management, Volume 5, Number 1, pages 50-56

[6] Othman, Zalinda, Subari, Khairanum, Morad, Norashima, Applications of Fuzz Inference Systems and Genetic Algorithms in Integrated Process Planning and Scheduling www.journal.au.edu/ijcim/2002/may02/article5.doc

[7] Rangarajan, S (2007), "Synchronous and Asynchronous Education Delivery", On-line Journal of Space Communication article can be found here -- http://satjournal.tcom.ohiou.edu/

[8] Samarajiva, Rohan. (2005). Mobilizing information and communications technologies for effective disaster warning: lessons from the 2004 tsunami. New Media and Society, 7 (6), 731-747.

[9] N. Waidyanatha, G. Gow, P. Anderson (2007). "Hazard Warnings in Sri Lanka: Challenges of Internetworking with Common Alerting Protocol". Proceedings of the ISCRAM Conference, Delft, Netherlands, May 2007. (In Press)

[10] N. Waidyanatha, G. Gow, P. Anderson (2007 August). "Common Alerting Protocol Message Broker for Last-Mile Hazard Warnings in Sri Lanka: An Essential Component", Proceedings of the $2^{\text {nd }}$ International ISCRAM Workshop, Harbin, China. (In Press) 